\title{
Sub-parts per billion detection of trace volatile chemicals in human breath using Selected Ion Flow Tube Mass Spectrometry Brian M Ross
}

\author{
Address: Northern Ontario School of Medicine, Departments of Biology and Chemistry, and the Public Health Program, Lakehead University, \\ Thunder Bay, Ontario, Canada \\ Email: Brian M Ross - brian.ross@normed.ca
}

Published: 10 July 2008

BMC Research Notes 2008, I:4I doi:10.| |86/1756-0500-I-4|

This article is available from: http://www.biomedcentral.com/I756-0500/I/4I

(c) 2008 Ross; licensee BioMed Central Ltd.

This is an Open Access article distributed under the terms of the Creative Commons Attribution License (http://creativecommons.org/licenses/by/2.0), which permits unrestricted use, distribution, and reproduction in any medium, provided the original work is properly cited.
Received: 22 April 2008

Accepted: 10 July 2008

\begin{abstract}
Background: Selected ion flow tube mass spectrometry (SIFT-MS) allows the real time quantification of trace gases in air. Due to its tolerance of high humidity levels the technique is particularly suited to the chemical analysis of breath. The detection limit of SIFT-MS has previously reported to be approximately 5 - 10 PPBV which is insufficient for the measurement of some low abundance constituents of breath. Recent developments in the design of SIFT-MS instruments have increased the ion precursor count rates. It is, however, unclear as to how these advances will affect instrument sensitivity for breath analysis.
\end{abstract}

Findings: Standard gases were prepared by adding known quantities of compounds present at zero or very low levels in breath (xylene and toluene) to either humidified bottled air or actual human breath. These were then analysed by SIFT-MS to calculate the limits of detection for each compound under conditions which mimic a single breath exhalation. For xylene and toluene the limits of detection was approximately 0.5 PPBV per 10 seconds of analysis time. Results gained using this level of sensitivity suggested the presence of low levels of the compounds indole and methylindole in human alveolar and static oral air, although further studies are necessary to confirm these findings.

Conclusion: Recent advances in SIFT-MS have increased the techniques sensitivity for breath analysis into the sub PPBV range enabling the real time quantification of low level trace gases in human breath.

\section{Background}

The measurement of volatile gases in breath for the purpose of the diagnosis, screening and monitoring of disease is an attractive proposition due to the inherently noninvasive nature of such methodology [1]. For example, the volatile biomarker nitric oxide has been successfully developed for the monitoring of airway inflammation while other putative markers for a conditions including cancer have been identified [2]. To be adopted clinically the methods used for the detection and quantification of breath biomarkers likely need to be rapid, simple to use, reproducible and able to detect the presence of disease early in its course. The latter feature can reduce mortality, morbidity, patient distress and decrease associated costs for the healthcare system [3]. As such more sensitive detection methods may offer the advantage that volatile biomarkers can be detected and acted upon at earlier stages in the disease process. 
A technique which shows promise for diagnostic breath analysis is Selected Ion Flow Tube Mass Spectrometry (SIFT-MS). SIFT-MS is suited to this role due to its linear response, reproducible absoloute concentration measurements, rapid analysis and lack of interference by the major constituents of expired air, in particular water vapour [4]. SIFT-MS is a form of chemical ionization in which precursor ions (usually $\mathrm{H}_{3} \mathrm{O}^{+}, \mathrm{NO}^{+}$or $\mathrm{O}_{2}^{+}$) are reacted with gas mixtures to produce ionized products characteristic of the volatile chemicals present [4]. The specific precursor ions used in the reaction, formed using a microwave water vapour ion source, are selected using a quadrupole mass filter. The precursor ions are then introduced into a fast flowing stream of helium and reacted with the air samples containing the trace gases of interest in a short flow tube. The ionized products along with remaining precursor ions are then quantified using a downstream quadrupole mass analyser. Once the rate constant for the reaction is known absolute concentrations of the trace gases present can be calculated without the need for calibration standards.

The sensitivity of SIFT-MS instruments has been a limiting factor since many potential biomarkers are present at concentrations in the low parts per billion by volume (PPBV) range or lower. This abundance is currently considered to be at or beneath the limits of detection for the technique, reported to be in the single digit parts per billion range for a single breath (10 second measurement time) [4-6]. The sensitivity of SIFT-MS instruments is partly dependent on the rate constant for the reaction between the precursor ion and the trace gas but also on several modifiable factors: the rate of precursor ion production, the amount of trace gas introduced in a given time, the measurement time and the background 'noise' signal produced in the absence of analyte. Recently it has been reported [7] that doubling the sampling flow rate allowed the detection of phosphine gas at concentrations of approximately 200 parts per trillion by volume (PPTV). Whether such sensitivities can be achieved for breath analysis by this modification is unclear since the authors used dry nitrogen as a carrier gas rather than the humid air mixture characteristic of human breath [7]. This difference is of importance since increasing the sampling flow rate of expired breath elevates the proportion of precursor ions reacting with the abundant water vapour thereby potentially antagonising the beneficial effect of the increased sampling rate [4]. Alternatively, a recent report suggests that optimizing the water content of the gas mixture used in the instrument's ion source can markedly elevates the rate of precursor ion generation. Using this modification the effect of enhanced precursor rates upon instrument sensitivity for the analysis of humid air mixtures has been investigated.

\section{SIFT-MS analysis}

SIFT-MS analysis was performed using a Profile 3 instrument (Instrument Science, UK) modified to allow a varia- ble water vapour abundance to enter the microwave source as described [8]. By this means $\mathrm{H}_{3} \mathrm{O}^{+}$count rates of approximately $2.2 \times 10^{6}$ counts per second (cps) were achieved while introducing ambient air in the SIFT-MS, with the total count rate of $\mathrm{H}_{3} \mathrm{O}^{+}$plus it's hydrates $\left(\mathrm{H}_{3} \mathrm{O}^{+} \cdot\left(\mathrm{H}_{2} \mathrm{O}\right)_{\mathrm{n}}\right.$ where $\mathrm{n}=1,2$ or 3$)$ being approximately $2.5 \times 10^{6} \mathrm{cps}$; typical maximal $\mathrm{O}_{2}{ }^{+}$count rates were approximately $3 \times 10^{6} \mathrm{cps}$ while $\mathrm{NO}^{+}$were approximately $1.8 \times 10^{6} \mathrm{cps}$.

\section{Gas standards prepared with humidified bottled gas}

For these experiments the compounds xylene and toluene (Sigma Aldrich, USA) were chosen since both are present in low levels in the breath of healthy non-smokers (BRoss, unpublished observations). A 5 L Tedlar bag (SKC Inc, USA) was filled and evacuated 3 times with bottled air to reduce the concentration of bag derived volatile compounds. The bag was then inflated with bottled air before followed by the introduction of $20 \mathrm{~mL}$ of water into the bag via the sampling valve port. The bag was then heated to $40^{\circ} \mathrm{C}$ for 30 minutes to produce humidified air which was used in subsequent experiments. A measured quantity $(260 \mathrm{~mL})$ of the humidified air was then transferred to empty $0.5 \mathrm{~L}$ Tedlar bags by means of a heated syringe. Up to $500 \mu \mathrm{L}$ of a commercially available gas standard containing approximately 5 parts per million by volume xylene and toluene in helium (Standard and Technical Gases, United Kingdom) was then introduced into the 0.5 L bags using 50, 250 or $500 \mu \mathrm{l}$ capacity gas syringes (Fisher Scientific, Canada) via a septum to produce known concentrations of each gas. After further incubation at $40^{\circ} \mathrm{C}$ for 15 minutes to allow mixing, the standards were introduced into the SIFT-MS by negative pressure via a transfer line heated to $65^{\circ} \mathrm{C}$ at a flow rate of 0.21 Torr $\mathrm{L} \mathrm{s}^{-1}$. Measurements were made by opening the inlet valve and waiting 10 seconds before quantifying gas levels over the period 10 to 20 seconds.

The water content of the gas samples prepared using humidified bottled air $(5.8 \pm 0.1 \%$ (mean \pm SD) was similar to that of human breath. The concentration of xylene and toluene (measured by their reaction with the $\mathrm{H}_{3} \mathrm{O}^{+}$ precursor as previously described in detail [9]) in humidified bottled air prior to introduction of these chemicals was not differentiable from the instrument background i.e. that derived from the count rate determined in the absence of sampled air. Using a 10 second sampling period both xylene and toluene could be differentiated from background at concentrations of approximately 500 PPTV (Figure 1) with instrument blanks (sampling line closed) being approximately $0.5 \mathrm{cps}$ for both compounds, while 500 PPTV resulted in approximately 2 cps product ions. Increasing concentrations of both compounds produced a linear rise in measured concentration over the concentration investigated (approximately 0 - 8 PPBV). 

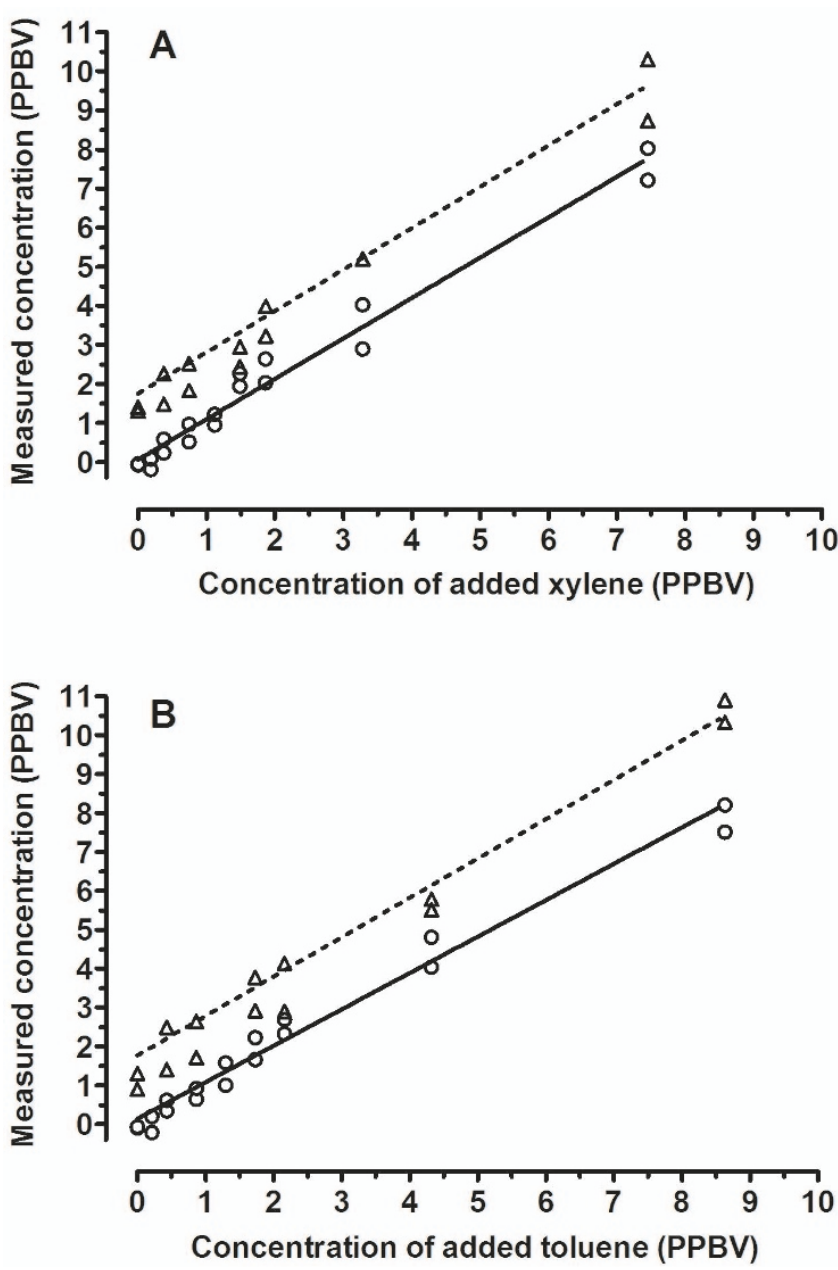

Figure I

Detection of xylene and toluene added to humidified air and human breath. Xylene (A) or toluene (B) vapour were added to either humidified bottled air (circles) or human breath (triangles) contained in Tedlar bags to produce a range of concentrations in duplicate. The standards were introduced into the sampling line of the SIFT-MS and after a delay of 10 seconds xylene and toluene levels were measured by reaction with the $\mathrm{H}_{3} \mathrm{O}^{+}$precursor for a period of $10 \mathrm{sec}-$ onds. These values were then plotted against the calculated standard concentrations. The best fit linear regression line is shown for both bottled air (solid line) and human breath (dashed line).

\section{Gas standards prepared using human breath}

A breath sample was obtained from a 40 year old male in good health followed by the construction of gas standards using a similar method as for bottled air with the exception that no humidification was required. Specifically, the subject exhaled into a $3 \mathrm{~L}$ Tedlar bag and $260 \mathrm{~mL}$ was transferred into $0.5 \mathrm{~L}$ Tedlar bags, followed by the addition of known quantities of the 5 PPMV gas standard in helium by means of a gas syringe. The subject gave informed consent for the procedure. Detectable concentrations of xylene (product ion $\mathrm{m} / \mathrm{z} 107, k=2.3 \times 10^{-9} \mathrm{~cm}^{3}$ $\mathrm{s}^{-1}$ ) and toluene (product ion $\mathrm{m} / \mathrm{z} 93, k=2.1 \times 10^{-9} \mathrm{~cm}^{3} \mathrm{~s}^{-}$ 1) product ions, measured using the $\mathrm{H}_{3} \mathrm{O}^{+}$precursor, were present in the absence of added standards (approximately 1 PPBV for xylene and toluene). These ions could well be derived from other compounds, however levels measured were significantly above instrument backgrounds (levels recorded in the absence of sampled air) and of similar concentrations (approximately 1 - 2 PPBV) when xylene or toluene levels were measured using reaction with the $\mathrm{NO}^{+}$or $\mathrm{O}_{2}{ }^{+}$precursors (xylene: product ion $\mathrm{m} / \mathrm{z} 106, k=$ $1.4 \times 10^{-9} \mathrm{~cm}^{3} \mathrm{~s}^{-1}$; toluene: product ion $\mathrm{m} / \mathrm{z} 92, k=1.4 \times$ $10^{-9} \mathrm{~cm}^{3} \mathrm{~s}^{-1}$ ). It should be noted that the proton bound dimer of ethanol has the same $\mathrm{m} / \mathrm{z}$ as the product of the reaction between toluene and $\mathrm{H}_{3} \mathrm{O}^{+}$. As such, in the presence of high ethanol concentrations ethanol may contribute to the apparent toluene signal making the use of the other precursor ions advisable for the analysis of this compound. It is presently unclear as to whether these xylene and toluene are compounds produced metabolically or are due to ambient levels of these gases which were present in the environment at similar concentrations. Using a 10 second sampling period, 500 PPTV exogenous concentrations of both compounds were differentiable from the exhaled breath levels (Figure 1).

\section{Measurement of trace malodorous compounds in human breath}

In order to further investigate the ability of SIFT-MS to detect compounds present at concentrations of approximately 1 PPBV or less alveolar breath analysis was performed using three human subjects (a 40 year old female, a 41 year old male and a 26 year old female all of whom gave informed consent). Alveolar breath and static oral air were sampled as described [10]. Briefly, to sample alveolar breath the subject expired via a heated stainless steel tube into which was inserted the SIFT-MS sampling line so that a small fraction of the flow was drawn into the instrument using negative pressure at a flow rate of 0.21 Torr $\mathrm{L}$ $\mathrm{s}^{-1}$. For oral air the stainless steel sampling tube was sealed at one end and an adapter attached at the other end which allows a piece of PTFE tubing (length $10 \mathrm{~cm}$, OD 1/4", ID $3 / 8$ ") to be attached. The subject was asked to close their mouth and breathe though their nose for 3 minutes to concentrate volatile compounds in the mouth. A piece of tubing is then inserted $2.5-5 \mathrm{~cm}$ into a nearly closed mouth. The subject is asked to not touch any mouth surface with the tube, and must not blow into, or inhale through, the tubing. Levels of the test compounds were measured using the $\mathrm{H}_{3} \mathrm{O}^{+}$precursor by their following product ions and utilising the following rate constants: hydrogen sulphide: $\mathrm{m} / \mathrm{z} 35, k=2.0 \times 10^{-9} \mathrm{~cm}^{3} \mathrm{~s}^{-1}$; methylmercaptan: $\mathrm{m} / \mathrm{z} 49, k=2.5 \times 10^{-9} \mathrm{~cm}^{3} \mathrm{~s}^{-1}$; indole: $\mathrm{m} / \mathrm{z} 118$ and $136, k=3.3 \times 10^{-9} \mathrm{~cm}^{3} \mathrm{~s}^{-1}$; and methylindole: $\mathrm{m} / \mathrm{z} 132$ 
and $150, k=3.3 \times 10^{-9} \mathrm{~cm}^{3} \mathrm{~s}^{-1}[10-12]$. As expected [10] (Table 1) oral air contained significant levels of the sulphur compounds hydrogen sulphide and methylmercaptan while levels in alveolar air were very low. For methylmercaptan the actual alveolar concentrations of the gas may be lower given that the reaction of $\mathrm{H}_{3} \mathrm{O}^{+}$with the ${ }^{18} \mathrm{O}$ isotopologue of ethanol will result in the production of the same product ion as that with methylmercaptan. As such approximately $0.6 \%$ of the ethanol concentration would contribute to the apparent methylmercaptan level measured using the above procedure e.g. 1 PPMV ethanol would contribute 6 PPB to the methylmercaptan concentration. Given the ubiquity of ethanol in both laboratory and clinical settings this mimicking effect of the compound should always be taken into account. The enhanced sensitivity of the SIFT-MS also allowed the detection ion product signatures of the compounds indole and methylindole. The presence of these compounds in human breath was supported by the generation of product ions of m/z 117 and 131 at rates approximately 2 cps above background for the reaction of human breath with $\mathrm{O}_{2}{ }^{+}$, which are consistent with products generated by charge transfer reactions with indole and methylindole respectively [12]. Such rates of product ion generation equate with similar concentration levels of the compounds with that detected using $\mathrm{H}_{3} \mathrm{O}^{+}$. The possibility that these ion products could be due to isopotopologues must be considered although significant generation of ions one or two mass units lower that the putative product ions for methylindole and indole was not apparent in the subjects studied. Nevertheless, as the sensitivity SIFT-MS increases the possibility that the presence of isopotopologues will become a significant factor in any particular measurement is also increased. In addition, the introduction of humidified lab air possessing a similar water content to that of human breath $(6 \% \mathrm{v} / \mathrm{v})$ did not change the apparent ambient levels of these compounds, suggesting that the product ions concerned are not due to a hydrate of a reaction product derived from another compound. The presented data therefore are suggestive of both methylindole and indole being present in human breath alve- olar breath at low levels. Both compounds are characteristic of faecal odour and their detection in breath at low levels may derive from either the gut or from an oral bacterial source. Further investigation is however required to conclusively determine the existence of the indoles in human breath, particularly as the construction of accurate spectra is made difficult due to their apparent low abundance.

\section{Limits of detection and quantification}

The presented data suggest that recent advances in the design of the ion source intrinsic to SIFT-MS instruments can be utilized to achieve significant sensitivity gains for breath trace gas analysis. The rate of precursor ion generation reported in this and another recent study [8] are in the 2 - 3 million counts per second range for $\mathrm{H}_{3} \mathrm{O}^{+}$, a rate approaching the 10 million counts per second generated by a related technique, proton transfer mass spectrometry (PTR-MS) [13]. The ion generation method used by SIFTMS allows the selection of the alternative $\mathrm{NO}^{+}$or $\mathrm{O}_{2}{ }^{+}$precursor ions to increase the chemical resolution of SIFT-MS compared to PTR-MS, while avoiding the quantification errors encountered by PTR-MS occurring due to the variably elevated ion energies typical of the technique [14]. By optimising the water vapour content of the ion source gas mixture the precursor count rates were sufficient to allow the detection of analyte concentrations of approximately 500 PPTV using the $\mathrm{H}_{3} \mathrm{O}^{+}$precursor. This was achieved using humidified air samples similar to human breath with a sampling time typical of a single expiration (10 seconds).

The determination of the limit of detection (LOD) can also be estimated using various parameters associated with the SIFT-MS analysis [7], these being the sensitivity of the measurement (how many product ions are produced for a given concentration of analyte in a particular time) and the instrument background counts at the product $\mathrm{m} / \mathrm{z}$, that is the counts produced in the absence of analyte. For the compounds measured in this study sensitivity was in the range 2 - 4 cps per PPBV, while the background

Table I: Levels of trace volatile compounds in alveolar and oral air.

\begin{tabular}{|c|c|c|c|c|c|}
\hline Sample & Compartment & Indole & Methylindole & Hydrogen Sulphide & Methylmercaptan \\
\hline Ambient & NA & 0.4 & 0 & 0.4 & 4 \\
\hline \multirow[t]{2}{*}{ Subject I } & Alveolar & 1.7 & 0.4 & 10 & 10 \\
\hline & Oral & 2.4 & 0.8 & 48 & 71 \\
\hline \multirow[t]{2}{*}{ Subject 2} & Alveolar & 2.2 & 0.4 & 9.2 & 9.4 \\
\hline & Oral & 3.2 & 0.9 & 23 & 44 \\
\hline \multirow[t]{2}{*}{ Subject 3} & Alveolar & 1.1 & 0.9 & II & 4.3 \\
\hline & Oral & 1.7 & 1.2 & $4 I$ & 38 \\
\hline
\end{tabular}

Levels of indole, methylindole (all isomers), hydrogen sulphide and methylmercaptan were measured in ambient air, or in a single alveolar breath or static oral air (approximately 10 second measurement duration) of 3 healthy human subjects by SIFT-MS using characteristic products ions of the reaction with $\mathrm{H}_{3} \mathrm{O}^{+}$precursors. Values are in PPBV. NA: not applicable. 
count rates for the product(s) ions were in the range 0.3 to 3 counts per second. The estimated LOD for 10 second measurements were calculated as 170, 130, 300, 440, 320 and 350 PPTV for indole, methylindole, hydrogen sulphide, methylmercaptan, xylene and toluene respectively. For the latter two compounds such computed values are in agreement with empirically derived limits of detection (see Figure 1). It is also possible to calculate the limit of quantification (LOQ), the concentration which can be determined with a particular level of precision. For a standard deviation of $20 \%$ using a 10 second measurement time the LOQs are 0.8, 0.7, 1.0, 1.3, 1.4 and 1.5 PPBV for the presumed analysis of indole, methylindole, hydrogen sulphide, methylmercaptan, xylene and toluene respectively. As such the presented data indicate that the modified instrument used in this study can produce reasonably precise measurements of each gas present at a concentration of approximately $1 \mathrm{PPBV}$. Clearly the technique is capable of lower LOD and LOQ when longer measurement times are used, but this will necessarily result in an inability to quantify trace gases present at these concentrations in a single expiration. The use of a breath storage device e.g. Tedlar bags, can allow longer measurement times to be used although this complicates the sampling methodology and can be the source of artefact.

\section{Conclusion}

In conclusion, advances in ion source design have resulted in significant improvements in the sensitivity of the SIFTMS procedure resulting in the potential for additional biomarkers to be readily investigated. As the ion generation process becomes better understood it is likely that additional improvements in the rate of precursor ion generation will be achieved with a concomitant enhancement of sensitivity. It should be recognized, however, that background count rates also put a limit on the trace gas concentration that can be detected in a given time period. Background counts are influenced by a variety of factors including electrical noise in the detector, impurities in the carrier helium gas, the presence of isotopologues and hydrates of other compounds, and by the production of low abundance ions in the source which possess the same $\mathrm{m} / \mathrm{z}$ ratio as the reaction product of the compound of interest [15]. The two latter factors may ultimately limit the detection sensitivity of SIFT-MS for specific trace gases rather than the rate of precursor ion generation. Optimisation of the ion source and/or the upstream mass filter to reduce the introduction of unwanted ions into the flow tube may, however, alleviate the problem of unwanted low abundance source ions. Nevertheless, for many trace gases sub-PPBV detection in single breath samples by SIFT-MS is readily achievable with the likelihood of further sensitivity enhancements in the future.

\section{Competing interests}

The author declares that they have no competing interests.

\section{Authors' contributions}

All experimental work and production of the manuscript was by BMR.

\section{Acknowledgements}

This work was aided by a grant from the Northern Cancer Research Foundation to BMR.

\section{References}

I. Amann A, ŠpanEl P, Smith D: Breath analysis: the approach towards clinical applications. Mini Rev Med Chem 2007, 7:115-129.

2. Katial R, Stewart L: Exhaled nitric oxide: a test for diagnosis and control of asthma? Curr Allergy Asthma Rep 2007, 7:459-463.

3. Fields MM, Chevlen E: Screening for disease: making evidencebased choices. Clin J Oncol Nurs 2006, 10:73-76.

4. ŠpanEI P, Smith D: Selected ion flow tube mass spectrometry for on-line trace gas analysis in biology and medicine. Eur J Mass Spectrom 2007, I 3:77-82.

5. Lovett AM, Reid NM, Buckley JA, French JB, Cameron DM: Realtime analysis of breath using an atmospheric pressure ionization mass spectrometer. Biomed Mass Spectrom 1979, 6:91-97.

6. Skeldon KD, Gibson GM, Wyse CA, McMillan LC, Monk SD, Longbottom C, Padgett MJ: Development of high-resolution realtime sub-ppb ethane spectroscopy and some pilot studies in life science. Appl Opt 2005, 44:47| 2-472I.

7. Milligan DB, Francis GJ, Prince BJ, McEwan MJ: Demonstration of selected ion flow tube MS detection in the parts per trillion range. Anal Chem 2007, 79:2537-2540.

8. ŠpanEl O, Dryahina K, Smith D: Microwave plasma ion sources for selected ion flow tube mass spectrometry: Optimizing their performance and detection limits for trace gas analysis. Int J Mass Spectrom 2007, 267: I 17-124.

9. Ross BM, Vermeulen N: The combined use of thermal desorption and selected ion flow tube mass spectrometry for the quantification of xylene and toluene in air. Rapid Commun Mass Spectrom 2007, 21:3608-3612.

10. Ross BM, Dadgostar N, Bloom M, McKeown L: The analysis of oral air using selected ion flow tube mass spectrometry in persons with and without a history of oral malodour. Int J Dent Hyg in press.

II. ŠpanEI P, Smith D: Quantification of hydrogen sulphide in humid air by selected ion flow tube mass spectrometry. Rapid Commun Mass Spectrom 2000, I 4: I I 36-I I 40.

12. Wanga T, ŠpanEI P, Smith D: A selectedion flow tube, SIFT, study of the reactions of $\mathrm{H}_{3} \mathrm{O}^{+}$, $\mathrm{NO}^{+}$and $\mathrm{O}_{2}{ }^{+}$ions with several $\mathrm{N}$ - and O-containing heterocyclic compounds in support of SIFT-MS. Int J Mass Spectrom 2004, 237:167-174.

13. Hewitt $\mathrm{CN}$, Hayward S, Tani A: The application of proton transfer reaction-mass spectrometry (PTR-MS) to the monitoring and analysis of volatile organic compounds in the atmosphere. J Environ Monit 2003, 5:1-7.

14. ŠpanEI P, Smith D: The challenge of breath analysis for clinical diagnosis and therapeutic monitoring. Analyst 2007, 132:390-396.

15. ŠpanEI P, Hall EFH, Workman CT, Smith D: A directly coupled monolithic rectangular resonator forming a robust microwave plasma ion source for SIFT-MS. Plasma Sources Sci Technol 2004, 1 3:282-284. 